Received by the editors: May 19, 2020; Accepted: October 26, 2020

\title{
ON THE CHINESE CHECKERS SPHERICAL INVERSIONS IN THREE DIMENSIONAL CHINESE CHECKERS SPACE
}

\author{
Adnan PEKZORLU and Ayşe BAYAR \\ Osmangazi University Department of Mathematics-Computer, 26480 Eskişehir, TURKEY
}

\begin{abstract}
In this paper, we study an inversion with respect to a Chinese checkers sphere in the three dimensional Chinese Checkers space, and prove several properties of this inversion. We also study cross ratio, harmonic conjugates and the inverse images of lines, planes and Chinese Checkers spheres in three dimensional Chinese Checkers space.
\end{abstract}

\section{INTRODUCTION}

In the game of Chinese checkers, checkers are allowed to move in the vertical (north and south), horizontal (east and west), and diagonal (northeast, northwest, southeast and southwest) directions. In [7, Krause asked how to develop a distance function that measures and made a suggestion for the idea of Chinese Checkers geometry and in G. Chen, introduced it by defining the metric in the coordinate plane 22 . The inversion was introduced by Perga and then studied and applied by Steiner about 1820s 2. During the following decades, many physicists and mathematicians independently rediscovered inversions, proving the properties that were most useful for their particular applications (for some references see [1], [4, [10]). Many kinds of generalizations of inversion transform have been presented in literature. The inversions with respect to the central conics in real Euclidean plane was introduced in 3. Then the inversions with respect to ellipse was studied detailed in 13. In three-dimensional space a generalization of the spherical inversion is given in 16]. Also, the inversions with respect to the taxicab distance, alpha-distance [17], 21], [19] or in general a p-distance [20]. The circle inversion have been generalized in three-dimensional space by using a sphere as the circle of inversion [16].

2020 Mathematics Subject Classification. 40E99, 51F99, 51B20, 51K99.

Keywords and phrases. Chinese checkers space, Chinese checkers sphere, inversion, harmonic conjugates.

\adnan.pekzorlu@hotmail.com-Corresponding author; akorkmaz@ogu.edu.tr

(D) 0000-0001-9724-4084; 0000-0002-2210-5423.

(C)2020 Ankara University Communications Faculty of Sciences University of Ankara-Series A1 Mathematics and Statistics 
H. Minkowski was one of the developers in "non-Euclidean" geometry and found taxicab geometry [9]. The taxicab geometry has been studied and improved by some mathematicians (for some references see [5], 6], 8], [14, [18]).

An inversion in a sphere is a transformation of the space that flips the sphere inside-out. That is, points outside the sphere get mapped to points inside the sphere, and points inside the sphere get mapped outside the sphere. In the present article, we define a notion of inversion valid in three dimensional Chinese Checkers space. In particular, we define an inversion with respect to a Chinese Checkers sphere and prove several properties of this new transformation. Also we introduce inverse points, cross ratio, harmonic conjugates and the inverse images of lines, planes and Chinese Checkers spheres in three dimensional Chinese Checkers space.

\section{Chinese Checkers Spherical Inversions}

In this section, we introduce the inversion in a Chinese Checkers sphere.

The Chinese Checkers space $\mathbb{R}_{C}^{3}$ is almost the same as the Euclidean space $\mathbb{R}^{3}$. The points and lines are the same, and the angles are measured the same way, but the distance function is different. In $\mathbb{R}^{3}$ the Chinese Checkers metric is defined using the distance function

$$
d_{C}(A, B)=d_{L}(A, B)+(\sqrt{2}-1) d_{S}(A, B)
$$

where

$$
d_{L}(A, B)=\max \left\{\left|x_{1}-x_{2}\right|,\left|y_{1}-y_{2}\right|,\left|z_{1}-z_{2}\right|\right\}
$$

and

$$
d_{S}(A, B)=\min \left\{\left|x_{1}-x_{2}\right|+\left|y_{1}-y_{2}\right|,\left|x_{1}-x_{2}\right|+\left|z_{1}-z_{2}\right|,\left|y_{1}-y_{2}\right|+\left|z_{1}-z_{2}\right|\right\}
$$

where $A=\left(x_{1}, y_{1}, z_{1}\right), B=\left(x_{2}, y_{2}, z_{2}\right)$ in $\mathbb{R}^{3}$. The unit ball in $\mathbb{R}_{C}^{3}$ is the set of points $(x, y, z)$ in space which satisfy the equation

$$
\max \{|x|,|y|,|z|\}+(\sqrt{2}-1) \min \{|x|+|y|,|x|+|z|,|y|+|z|\}=1
$$

We can define the notion of inversion in $\mathbb{R}_{C}^{3}$ as an analogue of inversion in $\mathbb{R}^{3}$.

Definition 1. Let $\mathcal{S}$ be a Chinese Checkers sphere centered at a point $O$ with radius $r$ in $\mathbb{R}_{C}^{3}$. The inversion in the Chinese Checkers sphere $\mathcal{S}$ or the Chinese Checkers spherical inversion respect to $\mathcal{S}$ is the function such that

$$
I_{(O, r)}: \mathbb{R}_{C}^{3}-\{O\} \rightarrow \mathbb{R}_{C}^{3}-\{O\}
$$

defined by $I_{(O, r)}(P)=P^{\prime}$, for $P \neq O$ where $P^{\prime}$ is on the $\overrightarrow{O P}$ and

$$
d_{C}(O, P) \cdot d_{C}\left(O, P^{\prime}\right)=r^{2} .
$$

The point $P^{\prime}$ is said to be the Chinese Checkers spherical inverse of $P$ in $\mathcal{S}, \mathcal{S}$ is called the sphere of inversion and $O$ is called the center of inversion. 
The Chinese Checkers spherical inversions with respect to the sphere, like reflections, are involutions. The fixed points of $I_{(O, r)}$ are the points on the Chinese Checkers sphere $\mathcal{S}$ centered at $O$ with radius $r$.

Some basic properties about spherical inversion are given in the following items. Note that it is possible to extend every property of the Chinese Checkers circle inversion to Chinese Checkers spherical inversion.

Theorem 2. Let $\mathcal{S}$ be an Chinese Checkers sphere with the center $O$ in the Chinese Checkers spherical inversion $I_{(O, r)}$. If the point $P$ is in the exterior of $\mathcal{S}$ then the point $P^{\prime}$, the inverse of $P$, is interior to $\mathcal{S}$, and conversely.

Proof. Let the point $P$ be in the exterior of $\mathcal{S}$, then $d_{C}(O, P)>r$. If $P^{\prime}=I_{(O, r)}(P)$; then $d_{C}(O, P) \cdot d_{C}\left(O, P^{\prime}\right)=r^{2}$. Hence $r^{2}=d_{C}(O, P) \cdot d_{C}\left(O, P^{\prime}\right)>r \cdot d_{C}\left(O, P^{\prime}\right)$ and $d_{C}\left(O, P^{\prime}\right)<r$.

The inversion $I_{(O, r)}$ is undefined at the point $O$. However, we can add to the Chinese Checkers space a single point at infinite $O_{\infty}$, which is the inverse of the center $O$ of Chinese Checkers inversion sphere $\mathcal{S}$. So, the inversion $I_{(O, r)}$ is one-toone map of extended Chinese Checkers sphere.

Theorem 3. Let $\mathcal{S}$ be a Chinese Checkers sphere with the center $O=(0,0,0)$ and the radius $r$ in $\mathbb{R}_{C}^{3}$. If $P=(x, y, z)$ and $P^{\prime}=\left(x^{\prime}, y^{\prime}, z^{\prime}\right)$ are inverse points with respect to the Chinese Checkers spherical inversion $I_{(O, r)}$, then

$$
P^{\prime}=\frac{r^{2}}{\left(d_{C}(O, P)\right)^{2}} P
$$

Proof. The equation of $\mathcal{S}$ is $d_{C}(O, P)=d_{L}(O, P)+(\sqrt{2}-1) d_{S}(O, P)$. Suppose that $P=(x, y, z)$ and $P^{\prime}=\left(x^{\prime}, y^{\prime}, z^{\prime}\right)$ are inverse points with respect to the Chinese Checkers spherical inversion $I_{(O, r)}$. Since the points $O, P$ and $P^{\prime}$ are collinear and the rays $\overrightarrow{O P}$ and $\overrightarrow{O P^{\prime}}$ are same direction,

$$
\begin{gathered}
\overrightarrow{O P^{\prime}}=k \overrightarrow{O P}, k \in \mathbb{R}^{+} \\
\left(x^{\prime}, y^{\prime}, z^{\prime}\right)=(k x, k y, k z) .
\end{gathered}
$$

From $d_{C}(O, P) \cdot d_{C}\left(O, P^{\prime}\right)=r^{2}, k=\frac{r^{2}}{\left(d_{C}(O, P)\right)^{2}}$. Replacing the value of $k$ in $\left(x^{\prime}, y^{\prime}, z^{\prime}\right)=(k x, k y, k z)$, the equations of $x^{\prime}, y^{\prime}$ and $z^{\prime}$ are obtained.

Corollary 4. Let $\mathcal{S}$ be a Chinese Checkers sphere with the center $O=(a, b, c)$ and the radius $r$ in $\mathbb{R}_{C}^{3}$. If $P=(x, y, z)$ and $P^{\prime}=\left(x^{\prime}, y^{\prime}, z^{\prime}\right)$ are inverse points with respect to the Chinese Checkers spherical inversion $I_{(O, r)}$, then

$$
P^{\prime}-O=\frac{r^{2}}{\left(d_{C}(O, P)\right)^{2}}(P-O)
$$


Proof. Since the translation preserve distances in the Chinese Checkers space 15, 12] by translating in $\mathbb{R}_{C}^{3}(0,0,0)$ to $(a, b, c)$ one can easily get the value of $x^{\prime}, y^{\prime}$ and $z^{\prime}$.

Theorem 5. Let $P, Q$ and $O$ be any three collinear different points in $\mathbb{R}_{C}^{3}$. If the Chinese Checkers spherical inversion $I_{(O, r)}$ transform $P$ and $Q$ into $P^{\prime}$ and $Q^{\prime}$ respectively, then

$$
d_{C}\left(P^{\prime}, Q^{\prime}\right)=\frac{r^{2} d_{C}(P, Q)}{d_{C}(O, P) d_{C}(O, Q)} .
$$

Proof. Assume first that $O, P, Q$ are collinear. From the Definition 1 we conclude that $d_{C}(O, P) d_{C}\left(O, P^{\prime}\right)=r^{2}=d_{C}(O, Q) d_{C}\left(O, Q^{\prime}\right)$. Since the ratios of the Euclidean and Chinese Checkers distances along a line are same,

$$
\begin{aligned}
d_{C}\left(P^{\prime}, Q^{\prime}\right) & =\left|d_{C}\left(O, P^{\prime}\right)-d_{C}\left(O, Q^{\prime}\right)\right| \\
& =\left|\frac{r^{2}}{d_{C}(O, P)}-\frac{r^{2}}{d_{C}(O, Q)}\right| \\
& =\frac{r^{2} d_{C}(P, Q)}{d_{C}(O, P) d_{C}(O, Q)}
\end{aligned}
$$

is obtained.

When $O, P, Q$ are not collinear, the theorem is not valid in Chinese Checkers space, generally $\mathbb{R}_{C}^{3}$. For example, for $O=(0,0,0), P=(1,0,0), Q=(2,2,2)$ and $r=2 \sqrt{2}$, the inversion $I_{(O, r)}$ transform $P$ and $Q$ into $P^{\prime}=(8,0,0)$ and $Q^{\prime}=\left(\frac{4(9+4 \sqrt{2})}{45}, \frac{4(9+4 \sqrt{2})}{45}, \frac{4(9+4 \sqrt{2})}{45}\right)$. It follows that $d_{C}(O, P)=1$,

$d_{C}(O, Q)=4 \sqrt{2}-2$ and $d_{C}(P, Q)=3 \sqrt{2}-1, d_{C}\left(P^{\prime}, Q^{\prime}\right)=\frac{4(9+4 \sqrt{2})}{45}+8(\sqrt{2}-1)$.

Theorem 6. Let $P, Q$ and $O$ be any three non-collinear different points in $\mathbb{R}_{C}^{3}$ and $I_{(O, r)}$ be the inversion such that transform $P$ and $Q$ into $P^{\prime}$ and $Q^{\prime}$ respectively. If the direction of $P Q$ line is any element of $D$ sets $i=1,2,3$ such that

$$
D_{1}=\left\{u_{i} \mid i \in 1,2, \ldots, 18\right\}
$$

for $u_{1}=(1,0,0), u_{2}=(-1,0,0), u_{3}=(0,1,0), u_{4}=(0,-1,0), u_{5}=(0,0,1)$, $u_{6}=(0,0,-1), u_{7}=\left(\frac{1}{\sqrt{2}}, \frac{1}{\sqrt{2}}, 0\right), u_{8}=\left(-\frac{1}{\sqrt{2}},-\frac{1}{\sqrt{2}}, 0\right), u_{9}=\left(-\frac{1}{\sqrt{2}}, \frac{1}{\sqrt{2}}, 0\right)$, $u_{10}=\left(\frac{1}{\sqrt{2}},-\frac{1}{\sqrt{2}}, 0\right), u_{11}=\left(\frac{1}{\sqrt{2}}, 0, \frac{1}{\sqrt{2}}\right), u_{12}=\left(-\frac{1}{\sqrt{2}}, 0,-\frac{1}{\sqrt{2}}\right)$,$$
u_{13}=\left(-\frac{1}{\sqrt{2}}, 0, \frac{1}{\sqrt{2}}\right), u_{14}=\left(\frac{1}{\sqrt{2}}, 0,-\frac{1}{\sqrt{2}}\right), u_{15}=\left(0, \frac{1}{\sqrt{2}}, \frac{1}{\sqrt{2}}\right) \text {, }
$$$$
u_{16}=\left(0,-\frac{1}{\sqrt{2}},-\frac{1}{\sqrt{2}}\right), u_{17}=\left(0,-\frac{1}{\sqrt{2}}, \frac{1}{\sqrt{2}}\right), u_{18}=\left(0, \frac{1}{\sqrt{2}},-\frac{1}{\sqrt{2}}\right) \text {, }
$$$$
D_{2}=\left\{v_{i} \mid i \in 1,2, \ldots, 8\right\}
$$

for $v_{1}=\left(\frac{1}{2 \sqrt{2}-1}, \frac{1}{2 \sqrt{2}-1}, \frac{1}{2 \sqrt{2}-1}\right), v_{2}=\left(-\frac{1}{2 \sqrt{2}-1}, \frac{1}{2 \sqrt{2}-1}, \frac{1}{2 \sqrt{2}-1}\right)$, 


$$
\begin{gathered}
v_{3}=\left(\frac{1}{2 \sqrt{2}-1},-\frac{1}{2 \sqrt{2}-1}, \frac{1}{2 \sqrt{2}-1}\right), v_{4}=\left(\frac{1}{2 \sqrt{2}-1}, \frac{1}{2 \sqrt{2}-1},-\frac{1}{2 \sqrt{2}-1}\right), \\
v_{5}=\left(\frac{1}{2 \sqrt{2}-1},-\frac{1}{2 \sqrt{2}-1},-\frac{1}{2 \sqrt{2}-1}\right), v_{6}=\left(-\frac{1}{2 \sqrt{2}-1}, \frac{1}{2 \sqrt{2}-1},-\frac{1}{2 \sqrt{2}-1}\right), \\
v_{7}=\left(-\frac{1}{2 \sqrt{2}-1},-\frac{1}{2 \sqrt{2}-1}, \frac{1}{2 \sqrt{2}-1}\right), v_{8}=\left(-\frac{1}{2 \sqrt{2}-1},-\frac{1}{2 \sqrt{2}-1},-\frac{1}{2 \sqrt{2}-1}\right), \\
D_{3}=\left\{t_{i} \mid i \in 1,2, \ldots, 48\right\}
\end{gathered}
$$

$t_{1}=(1, \sqrt{2}+1,0), t_{2}=(1,-(\sqrt{2}+1), 0), t_{3}=(-1, \sqrt{2}+1,0)$,

$t_{4}=(-1,-(\sqrt{2}+1), 0), t_{5}=(1,0, \sqrt{2}+1), t_{6}=(1,0,-(\sqrt{2}+1))$,

$t_{7}=(-1,0, \sqrt{2}+1), t_{8}=(-1,0,-(\sqrt{2}+1)), t_{9}=(0, \sqrt{2}+1,1)$,

$t_{10}=(0,-(\sqrt{2}+1), 1), t_{11}=(0, \sqrt{2}+1,-1), t_{12}=(0,-(\sqrt{2}+1),-1)$,

$t_{13}=(0,1, \sqrt{2}+1), t_{14}=(0,1,-(\sqrt{2}+1)), t_{15}=(0,-1, \sqrt{2}+1)$,

$t_{16}=(0,-1,-(\sqrt{2}+1)), t_{17}=(\sqrt{2}+1,0,1), t_{18}=(-(\sqrt{2}+1), 0,1)$,

$t_{19}=(\sqrt{2}+1,0,-1), t_{20}=(-(\sqrt{2}+1), 0,-1), t_{21}=(\sqrt{2}+1,1,0)$,

$t_{22}=(-(\sqrt{2}+1), 1,0), t_{23}=(\sqrt{2}+1,-1,0), t_{24}=(-(\sqrt{2}+1),-1,0)$,

$t_{25}=(1, \sqrt{2}-1,0), t_{26}=(1,-(\sqrt{2}-1), 0), t_{27}=(-1, \sqrt{2}-1,0)$,

$t_{28}=(-1,-(\sqrt{2}-1), 0), t_{29}=(1,0, \sqrt{2}-1), t_{30}=(1,0,-(\sqrt{2}-1))$,

$t_{31}=(-1,0, \sqrt{2}-1), t_{32}=(-1,0,-(\sqrt{2}-1)), t_{33}=(0, \sqrt{2}-1,1)$,

$t_{34}=(0,-(\sqrt{2}-1), 1), t_{35}=(0, \sqrt{2}-1,-1), t_{36}=(0,-(\sqrt{2}-1),-1)$,

$t_{37}=(0,1, \sqrt{2}-1), t_{38}=(0,1,-(\sqrt{2}-1)), t_{39}=(0,-1, \sqrt{2}-1)$,

$t_{40}=(0,-1,-(\sqrt{2}-1)), t_{41}=(\sqrt{2}-1,0,1), t_{42}=(-(\sqrt{2}-1), 0,1)$,

$t_{43}=(\sqrt{2}-1,0,-1), t_{44}=(-(\sqrt{2}-1), 0,-1), t_{45}=(\sqrt{2}-1,1,0)$,

$t_{46}=(-(\sqrt{2}-1), 1,0), t_{47}=(\sqrt{2}-1,-1,0), t_{48}=(-(\sqrt{2}-1),-1,0)$

is valid.

$$
d_{C}\left(P^{\prime}, Q^{\prime}\right)=\frac{r^{2} d_{C}(P, Q)}{d_{C}(O, P) d_{C}(O, Q)}
$$

Proof. Since translations preserve the Chinese Checkers distances, it is enough to consider the center $O$ of the inversion sphere as the origin. Let $P, Q \in D_{1}$ in $\mathbb{R}_{C}^{3}$. If $P=(p, 0,0)$ and $Q=(0, q, 0)$, the images of $P$ and $Q$ respect to $I_{(O, r)}$ are $P^{\prime}=\left(\frac{r^{2}}{p}, 0,0\right)$ and $Q^{\prime}=\left(0, \frac{r^{2}}{q}, 0\right)$. It follows that

$$
\begin{gathered}
d_{C}\left(P^{\prime}, Q^{\prime}\right)=d_{L}\left(P^{\prime}, Q^{\prime}\right)+(\sqrt{2}-1) d_{S}\left(P^{\prime}, Q^{\prime}\right) \\
d_{L}\left(P^{\prime}, Q^{\prime}\right)=\max \left\{\left|\frac{r^{2}}{p}\right|,\left|\frac{r^{2}}{q}\right|, 0\right\} \\
d_{S}\left(P^{\prime}, Q^{\prime}\right)=\min \left\{\left|\frac{r^{2}}{p}+\frac{r^{2}}{q}\right|,\left|\frac{r^{2}}{p}\right|,\left|\frac{r^{2}}{q}\right|\right\}
\end{gathered}
$$


and then on $D_{1}$

$$
d_{C}\left(P^{\prime}, Q^{\prime}\right)=\frac{r^{2} d_{C}(P, Q)}{d_{C}(O, P) d_{C}(O, Q)} .
$$

If $P, Q \in D_{2}$ in $\mathbb{R}_{C}^{3}$. then the images of $P=\left(\frac{p}{2 \sqrt{2}-1}, \frac{p}{2 \sqrt{2}-1}, \frac{p}{2 \sqrt{2}-1}\right)$ and $Q=\left(-\frac{q}{2 \sqrt{2}-1}, \frac{q}{2 \sqrt{2}-1}, \frac{q}{2 \sqrt{2}-1}\right)$ respect to $I_{(O, r)}$ are $P^{\prime}=\left(\frac{r^{2}}{p(2 \sqrt{2}-1)}, \frac{r^{2}}{p(2 \sqrt{2}-1)}, \frac{r^{2}}{p(2 \sqrt{2}-1)}\right)$ and $Q^{\prime}=\left(-\frac{r^{2}}{q(2 \sqrt{2}-1)}, \frac{r^{2}}{q(2 \sqrt{2}-1)}, \frac{r^{2}}{q(2 \sqrt{2}-1)}\right)$.

It follows that

$$
\begin{aligned}
& d_{C}\left(P^{\prime}, Q^{\prime}\right)=d_{L}\left(P^{\prime}, Q^{\prime}\right)+(\sqrt{2}-1) d_{S}\left(P^{\prime}, Q^{\prime}\right) \\
& d_{L}\left(P^{\prime}, Q^{\prime}\right)=\max \left\{\left|\frac{r^{2}}{p(2 \sqrt{2}-1)}+\frac{r^{2}}{q(2 \sqrt{2}-1)}\right|,\left|\frac{r^{2}}{p(2 \sqrt{2}-1)}-\frac{r^{2}}{q(2 \sqrt{2}-1)}\right|\right\} \\
& d_{S}\left(P^{\prime}, Q^{\prime}\right)=\min \left\{\begin{array}{c}
\left|\frac{r^{2}}{p(2 \sqrt{2}-1)}+\frac{r^{2}}{q(2 \sqrt{2}-1)}\right|+\left|\frac{r^{2}}{p(2 \sqrt{2}-1)}-\frac{r^{2}}{q(2 \sqrt{2}-1)}\right|, \\
2\left|\frac{r^{2}}{p(2 \sqrt{2}-1)}-\frac{r^{2}}{q(2 \sqrt{2}-1)}\right|
\end{array}\right\}
\end{aligned}
$$

and then on $D_{2}$

$$
d_{C}\left(P^{\prime}, Q^{\prime}\right)=\frac{r^{2} d_{C}(P, Q)}{d_{C}(O, P) d_{C}(O, Q)} .
$$

If $P, Q \in D_{3}$ in $\mathbb{R}_{C}^{3}$. then the images of $P=(p, p(\sqrt{2}+1), 0)$ and $Q=(q,-q(\sqrt{2}+1), 0)$ respect to $I_{(O, r)}$ are $P^{\prime}=\left(\frac{r^{2}}{8 p}, \frac{r^{2}(\sqrt{2}+1)}{8 p}, 0\right)$ and $Q^{\prime}=\left(\frac{r^{2}}{8 q},-\frac{r^{2}(\sqrt{2}+1)}{8 q}, 0\right)$. So, we get

$$
\begin{gathered}
d_{C}\left(P^{\prime}, Q^{\prime}\right)=d_{L}\left(P^{\prime}, Q^{\prime}\right)+(\sqrt{2}-1) d_{S}\left(P^{\prime}, Q^{\prime}\right) \\
d_{L}\left(P^{\prime}, Q^{\prime}\right)=\max \left\{\left|\frac{r^{2}}{8 p}-\frac{r^{2}}{8 q}\right|,\left|\frac{r^{2}(\sqrt{2}+1)}{8 p}+\frac{r^{2}(\sqrt{2}+1)}{8 q}\right|, 0\right\} \\
d_{S}\left(P^{\prime}, Q^{\prime}\right)=\min \left\{\begin{array}{c}
\left|\frac{r^{2}}{8 p}-\frac{r^{2}}{8 q}\right|+\left|\frac{r^{2}(\sqrt{2}+1)}{8 p}+\frac{r^{2}(\sqrt{2}+1)}{8 q}\right|,\left|\frac{r^{2}}{8 p}-\frac{r^{2}}{8 q}\right|, \\
\left|\frac{r^{2}(\sqrt{2}+1)}{8 p}+\frac{r^{2}(\sqrt{2}+1)}{8 q}\right|
\end{array}\right\}
\end{gathered}
$$

and then on $D_{3}$

$$
d_{C}\left(P^{\prime}, Q^{\prime}\right)=\frac{r^{2} d_{C}(P, Q)}{d_{C}(O, P) d_{C}(O, Q)} .
$$




\section{Cross Ratio and Harmonic Conjugates}

The Chinese Checkers directed distance from the point $A$ to the point $B$ along a line $l$ in $\mathbb{R}_{C}^{2}$ is denoted by $d_{C}[A B]$. If the ray with initial point $A$ containing $B$ has the positive direction of orientation, $d_{C}[A B]=d_{C}(A, B)$ and if the ray has the opposite direction, $d_{C}[A B]=-d_{C}(A, B)$ [11]. The Chinese Checkers cross ratio is preserved by the inversion in the Chinese Checkers circle as in the taxicab plane in $[17$.

Now, we show the properties related to the Chinese Checkers cross ratio and harmonic conjugates in $\mathbb{R}_{C}^{3}$.

Definition 7. Let $A, B, C$ and $D$ be four distinct points on an oriented line in $\mathbb{R}_{C}^{3}$. We define the their Chinese Checkers cross ratio $(A B, C D)_{C}$ in $\mathbb{R}_{C}^{3}$ by

$$
(A B, C D)_{C}=\frac{d_{C}[A C]}{d_{C}[A D]} \frac{d_{C}[B D]}{d_{C}[B C]} .
$$

It is known that the cross ratio is positive if both $C$ and $D$ are between $A$ and $B$ or if neither $C$ nor $D$ is between $A$ and $B$, whereas the cross ratio is negative if the pairs $\{A, B\}$ and $\{C, D\}$ separate each other. Also, the cross ratio is an invariant under inversion in a sphere whose center is not any of the four points $A, B, C$ and $D$ in the taxicab plane, 11. Similarly, this property is valid in Chinese Checkers sphere.

Theorem 8. The inversion in a Chinese Checkers sphere in $\mathbb{R}_{C}^{3}$ preserves the Chinese Checkers cross ratio.

Proof. Let $A, B, C$ and $D$ be four collinear points on an oriented line $l$ with the center of the inversion $I_{(O, r)}$ in $\mathbb{R}_{C}^{3}$. Let $I_{(O, r)}$ transform $A, B, C$ and $D$ into $A^{\prime}, B^{\prime}, C^{\prime}$ and $D^{\prime}$, respectively. The Chinese Checkers spherical inversion reverses the Chinese Checkers directed distance from the point $A$ to the point $B$ along a line $l$ in $\mathbb{R}_{C}^{3}$ to the Chinese Checkers directed distance from the point $B^{\prime}$ to the point $A^{\prime}$ and preserves the separation or non separation of the pair $A, B$ and $C, D$. Hence it is suffices to show that $\left|\left(A^{\prime} B^{\prime}, C^{\prime} D^{\prime}\right)_{C}\right|=\left|(A B, C D)_{C}\right|$. This follows Theorem 2

$$
\begin{aligned}
\frac{d_{C}\left(A^{\prime}, C^{\prime}\right)}{d_{C}\left(A^{\prime}, D^{\prime}\right)} \frac{d_{C}\left(B^{\prime}, D^{\prime}\right)}{d_{C}\left(B^{\prime}, C^{\prime}\right)} & =\frac{\frac{r^{2} d_{C}(A, C)}{d_{C}(O, A) d_{C}(O, C)} \frac{r^{2} d_{C}(B, D)}{r^{2} d_{C}(A, D)} \frac{d_{C}(O, B) d_{C}(O, D)}{r^{2} d_{C}(B, C)}}{\frac{d_{C}(O, A) d_{C}(O, D)}{d_{C}(O, B) d_{C}(O, C)}} \\
& =\frac{d_{C}(A, C)}{d_{C}(A, D)} \frac{d_{C}(B, D)}{d_{C}(B, C)}
\end{aligned}
$$

Definition 9. Let $A$ and $B$ be two points on a line $l$ in $\mathbb{R}_{C}^{3}$, any pair $C$ and $D$ on the line l for which $\frac{d_{C}[A C]}{d_{C}[C B]}=\frac{d_{C}[A D]}{d_{C}[D B]}$ is said to divide $A$ and $B$ harmonically. The points 
$C$ and $D$ are called Chinese Checkers harmonic conjugates with respect to $A$ and $B$, and the Chinese Checkers harmonic set of points is denoted by $H(A B, C D)_{C}$.

It is clear that two distinct points $C$ and $D$ are Chinese Checkers harmonic conjugates with respect to $A$ and $B$ if and only if $(A B, C D)_{C}=-1$.

Theorem 10. Let $\mathcal{S}$ be a Chinese Checkers sphere with the center $O$, and the line segment $[A B]$ a diameter of $\mathcal{S}$ in $\mathbb{R}_{C}^{3}$. Let $P$ and $P^{\prime}$ be distinct points of the ray $O A$, which divide the segment $[A B]$ internally and externally. Then $P$ and $P^{\prime}$ are Chinese Checkers harmonic conjugates with respect to $A$ and $B$ if and only if $P$ and $P^{\prime}$ are inverse points with respect the Chinese Checkers spherical inversion $I_{(O, r)}$.

Proof. Suppose that $P$ and $P^{\prime}$ are Chinese Checkers harmonic conjugates with respect to $A$ and $B$ in $\mathbb{R}_{C}^{3}$. Then

$$
\begin{gathered}
\left(A B, P P^{\prime}\right)_{C}=-1 \\
\frac{d_{C}[A P]}{d_{C}\left[A P^{\prime}\right]} \frac{d_{C}\left[B P^{\prime}\right]}{d_{C}[B P]}=-1 .
\end{gathered}
$$

Since $P$ divides the line segment $[A B]$ internally and $P$ is on the ray $O B$,

$d_{C}(P, B)=r-d_{C}(O, P)$ and $d_{C}(A, P)=r+d_{C}(O, P)$. Since $P^{\prime}$ divides the line segment $[A B]$ externally and $P^{\prime}$ is on the ray $O B, d_{C}\left(A, P^{\prime}\right)=d_{C}\left(O, P^{\prime}\right)+r$ and $d_{C}\left(B, P^{\prime}\right)=d_{C}\left(O, P^{\prime}\right)-r$.

Hence

$$
\begin{gathered}
\frac{r+d_{C}(O, P)}{d_{C}\left(O, P^{\prime}\right)+r} \cdot \frac{d_{C}\left(O, P^{\prime}\right)-r}{d_{C}(O, P)-r}=-1 \\
\left(r+d_{C}(O, P)\right)\left(d_{C}\left(O, P^{\prime}\right)-r\right)=\left(d_{C}\left(O, P^{\prime}\right)+r\right) \cdot\left(r-d_{C}(O, P)\right) .
\end{gathered}
$$

Simplifying the last equality, $d_{C}(O, P) d_{C}\left(O, P^{\prime}\right)=r^{2}$ is obtained. Therefore $P$ and $P^{\prime}$ are Chinese Checkers inverse points with respect to the Chinese Checkers spherical inversion $I_{(O, r)}$.

Conversely, if $P$ and $P^{\prime}$ are Chinese Checkers inverse points with respect to the Chinese Checkers spherical inversion $I_{(O, r)}$, the proof is similar.

\section{Chinese Checkers Spherical Inversions of Lines, Planes and Chinese Checkers Spheres}

It is well known that inversions with respect to circle transform lines and circles into lines and/ or circles in Euclidean plane and Hyperbolic plane.

The following features are well known for inversion in Euclidean plane:

i) Lines passing through the inversion center are invariant.

ii) Lines that do not pass through the center of inversion transform circles passing through the center of inversion.

iii) Circles passing through the center of inversion transform lines does not pass through the center of the inversion.

iv) Circles not passing through the center of inversion transform circles does not pass through the center of the inversion. 
v) Circles with center of inversion transform circles with center of inversion.

In this section, we study the Chinese Checkers spherical inversion of lines, planes and Chinese Checkers spheres. The Chinese Checkers spherical inversion $I_{(O, r)}$ maps the lines, planes passing through $O$ onto themselves.

The Chinese Checkers spherical inversion $I_{(O, r)}$ maps Chinese Checkers spheres with centered $O$ onto Chinese Checkers spheres. But the Chinese Checkers spherical inversion of a sphere not passing through the centre of inversion is another Chinese Checkers sphere that does not contain the centre of inversion.

Theorem 11. Consider the inversion $I_{(O, r)}$ in a Chinese Checkers sphere $\mathcal{S}$ with the centre $O$. Every line and plane containing $O$ is invariant under the inversion.

Proof. It is clear that the straight lines containing $O$ onto themselves.

Let $\mathcal{S}$ be a Chinese Checkers sphere of inversion and $P=(x, y, z)$ with equation $d_{L}(O, P)+(\sqrt{2}-1) d_{S}(O, P)=r$ and the plane $M x+N y+T z=0$. Applying $I_{(O, r)}$ to this plane gives

$$
M \frac{r^{2} x^{\prime}}{\left(d_{C}(O, P)\right)^{2}}+N \frac{r^{2} y^{\prime}}{\left(d_{C}(O, P)\right)^{2}}+T \frac{r^{2} z^{\prime}}{\left(d_{C}(O, P)\right)^{2}}=0 .
$$

So, $M x^{\prime}+N y^{\prime}+T z^{\prime}=0$ is obtained.

The inverse of a plane not containing $O$ is not a Chinese Checkers sphere containing $O$.

Theorem 12. The inverse of a Chinese Checkers sphere with the centre $O$ with respect to the Chinese Checkers spherical inversion $I_{(O, r)}$ is a Chinese Checkers sphere containing $O$.

Proof. Since the translation preserve distance in $\mathbb{R}_{C}^{3}$, we can take a Chinese Checkers sphere $\mathcal{S}$ of inversion and $P=(x, y, z)$ with equation $d_{L}(O, P)+(\sqrt{2}-1) d_{S}(O, P)=r$ and $\mathcal{S}$ the Chinese Checkers sphere $d_{L}(O, P)+(\sqrt{2}-1) d_{S}(O, P)=k, k \in \mathbb{R}^{+}$. Applying $I_{(O, r)}$ to $\mathcal{S}$ gives

$$
d_{L}\left(O, P^{\prime}\right)+(\sqrt{2}-1) d_{S}\left(O, P^{\prime}\right)=\frac{r^{2}}{k} .
$$

Note that this is a Chinese Checkers sphere with the centre $O$.

Theorem 13. The inversion $I_{(O, r)}$ in a Chinese Checkers sphere $\mathcal{S}$ with centre $O$. Every edges, vertices and faces of Chinese Checkers sphere is invariant under the inversion.

Proof. The points of Chinese Checkers sphere are mapped by $I_{(O, r)}$ back onto Chinese Checkers sphere from the Definition 1. Hence every edges, vertices and faces of Chinese Checkers sphere is invariant under $I_{(O, r)}$. 


\section{REFERENCES}

[1] Blair, D., Inversion Theory and Conformal Mapping, Student Mathematical Library, American Mathematical Society, 9, 2000.

[2] Blåsjö Jakob, V., Steiner's Systematische Entwickelung: The Culmination of Classical Geometry, The Mathematical Intelligencer, 31(1) (2009), 21-29.

[3] Childress, N., Inversion with respect to the central conics, Mathematics Magazine, 38(3) (1965).

[4] Deza, E., Deza, M., Dictionary of Distances, Elsevier Science, 2006.

[5] Gelisgen, Ö., Kaya, R., The Taxicab Space Group, Acta Mathematica Hungarica, 122 (1) (2008), 187-200.

[6] Kaya, R., Akça, Z., Günaltıll, İ., Özcan, M., General Equation For taxicab Conics and Their Classification, Mitt. Math. Ges. Hamburg, 19 (2000), 135-148.

[7] Krause, E. F., Taxicab Geometry, Addison-Weley, Menlo Park, California,1975.

[8] Menger, K., You Will Like Geometry, Guildbook of Illinois Institute of Technology Geometry Exhibit, Museum of Science \& Industry,Chicago, Illinois, 1952.

[9] Minkowski, H., Gasammelte Abhandlungen, Chelsea Publishing Co., New York,1967.

[10] Nickel, J.A., A Budget of Inversion, Math. Comput. Modelling, 21(6) (1995), 87-93.

[11] Özcan, M., Kaya, R., On the Ratio of Directed Lengths in the Taxicab Plane and Related Properties, Missouri Journal of Mathematical Sciences, 14(2) (2002).

[12] Gelisgen, Ö., Kaya, R., Özcan, M., Distance Formulae in the Chinese Checker Space, Int.J. Pure Appl. Math., 26(1) (2006), 35-44.

[13] Ramirez, J.L., An Introduction to Inversion in an Ellipse, arXiv:1309.6378v1, Sept. 2013.

[14] Schattschneider, D. J., The Taxicab Group, American Mathematical Monthly, 91 (1984), 423-428.

[15] Kaya, R., Gelisgen, Ö., Bayar, A., Ekmekçi, S., Group of Isometries of CC-Plane, Missouri J. Math. Sci., 18 (2006), 221-233.

[16] Ramirez, J.L., Rubiano, G. N., A generalization of the spherical inversion, International Journal of Mathematical Education in Science and Technology, 48(1) (2016), 132-149.

[17] Bayar,A., Ekmekçi, S., On circular inversions in taxicab plane. J. Adv. Res. Pure Math., 6(4) (2014), 33-39.

[18] Akca, Z., Kaya, R., On the Distance Formulae In three Dimensional Taxicab Space, Hadronic Journal, 27 (2006), 521-532.

[19] Gelisgen, Ö., Ermiş, T., Some properties of inversions in alpha plane, Forum Geometricorum, 19 (2019), 1-9.

[20] Ramirez, J.L., Rubiano, G. N., Jurcic-Zlobec, B., Generating fractal patterns by using p-circle, Fractals, 23(4) (2015), 1-13.

[21] Pekzorlu, A., Bayar, A., Taxicab Spherical Inversions in Taxicab Space, Journal Of Mahani, Math. Research Center, 9(1-2) (2020), 45-54.

[22] Chen, G., Lines and Circles in Taxicab Geometry Master Thesis, Department of Mathematic and Computer Science, Central Missouri State Uni, 1992. 